\title{
Refining the anatomic boundaries of the endoscopic endonasal transpterygoid approach: the "VELPPHA area" concept
}

\author{
Ariel Kaen, MD, PhD, ${ }^{1}$ Eugenio Cárdenas Ruiz-Valdepeñas, MD, PhD, ${ }^{1}$ Alberto Di Somma, MD, ${ }^{2}$ \\ Francisco Esteban, MD, PhD, ${ }^{3}$ Javier Márquez Rivas, MD, PhD, ${ }^{1}$ and \\ Jesús Ambrosiani Fernandez, MD, PhD4
}

Departments of ${ }^{1}$ Neurosurgery and ${ }^{3}$ Otorhinolaryngology, Hospital Universitario Virgen del Rocío; ${ }^{4}$ Department of Anatomy and Human Embryology, University of Sevilla, Sevilla, Spain; and 'Division of Neurosurgery, School of Medicine and Surgery, Università degli Studi di Napoli Federico II, Naples, Italy

OBJECTIVE The endoscopic endonasal transpterygoid route has been widely evaluated in cadavers, and it is currently used during surgery for specific diseases involving the lateral skull base. Identification of the petrous segment of the internal carotid artery (ICA) is a key step during this approach, and the vidian nerve (VN) has been described as a principal landmark for safe endonasal localization of the petrous ICA at the level of the foramen lacerum. However, the relationship of the VN to the ICA at this level is complex as well as variable and has not been described in the pertinent literature. Accordingly, the authors undertook this purely anatomical study to detail and quantify the peri-lacerum anatomy as seen via an endoscopic endonasal transpterygoid pathway.

METHODS Eight human anatomical specimens (16 sides) were dissected endonasally under direct endoscopic visualization. Anatomical landmarks of the VN and the posterior end of the vidian canal (VC) during the endoscopic endonasal transpterygoid approach were described, quantitative anatomical data were compiled, and a schematic classification of the most relevant structures encountered was proposed.

RESULTS The endoscopic endonasal transpterygoid approach was used to describe the different anatomical structures surrounding the anterior genu of the petrous ICA. Five key anatomical structures were identified and described: the VN, the eustachian tube, the foramen lacerum, the petroclival fissure, and the pharyngobasilar fascia. These structures were specifically quantified and summarized in a schematic acronym-VELPPHA-to describe the area. The VELPPHA area is a dense fibrocartilaginous space around the inferior compartment of the foramen lacerum that can be reached by following the VC posteriorly; this area represents the posterior limits of the transpterygoid approach and, of utmost importance, no neurovascular structures were observed through the VELPPHA area in this study, indicating that it should be a safe zone for surgery in the posterior end of the endoscopic endonasal transpterygoid approach.

CONCLUSIONS The VELPPHA area represents the posterior limits of the endoscopic endonasal transpterygoid approach. Early identification of this area can enhance the safety of the endoscopic endonasal transpterygoid approach expanded to the lateral aspect of the skull base, especially when treating patients with poorly pneumatized sphenoid sinuses.

https://thejns.org/doi/abs/10.3171/2018.4.JNS173070

KEYWORDS skull base; transpterygoid approaches; endoscopic endonasal transpterygoid approach; pterygoid process; carotid artery; VELPPHA area; anatomy

$\mathrm{T}$ HE endoscopic endonasal transpterygoid approach is a lateral extension of the midline endonasal route; although it was initially described to access only the lateral recess of the sphenoid sinus, currently this route is used to reach the most lateral aspect of the skull base. In this surgical scenario, injury to the internal carotid artery (ICA) is one of the most feared complications.

The vidian nerve (VN) has been clearly described as a critical landmark for the safe identification of the petrous ICA in the foramen lacerum (FL) during the endoscopic

ABBREVIATIONS ET = eustachian tube; FL = foramen lacerum; ICA = internal carotid artery; VC = vidian canal; VELPPHA = vidian nerve, eustachian tube, foramen lacerum, petroclival fissure, and pharyngobasilar fascia; $\mathrm{VN}=$ vidian nerve.

SUBMITTED December 6, 2017. ACCEPTED April 5, 2018.

INCLUDE WHEN CITING Published online September 21, 2018; DOI: 10.3171/2018.4.JNS173070. 

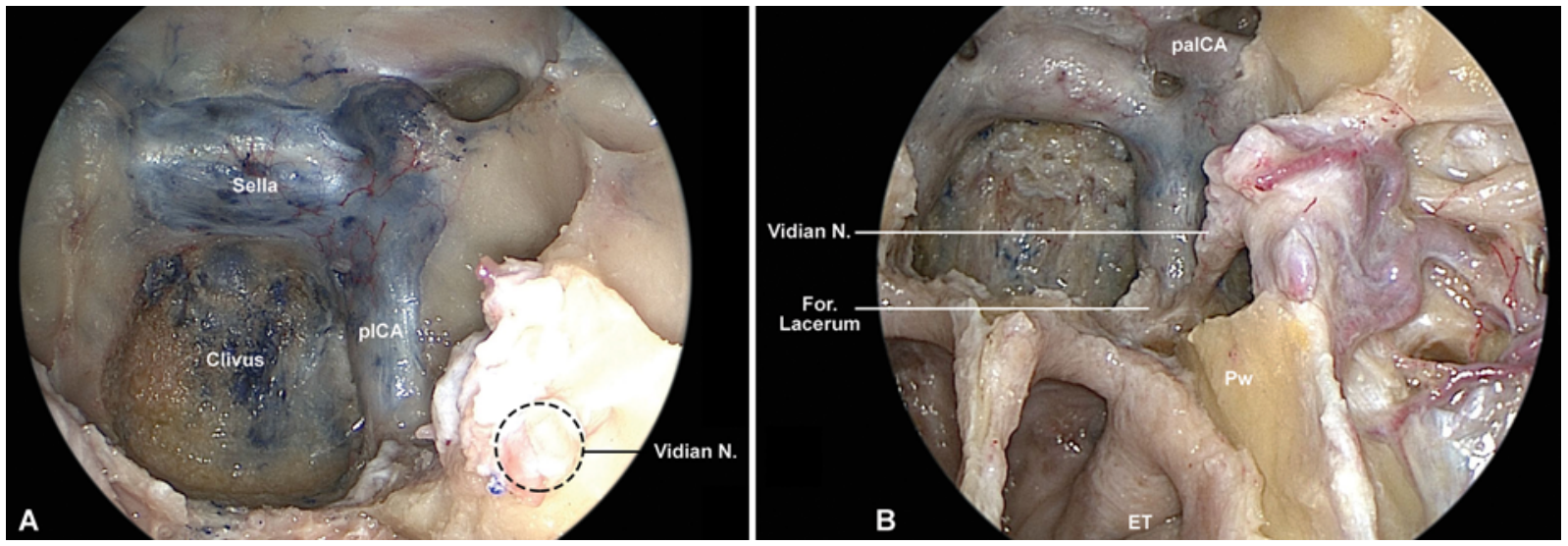

FIG. 1. Endonasal endoscopic view of the VN and VC during the initial steps of the approach $(\mathbf{A})$ and at a later phase $(\mathbf{B})$. ET = eustachian tube; For. = foramen; $\mathrm{N}$. = nerve; paICA = parasellar ICA; $\mathrm{pICA}=$ paraclival ICA; $\mathrm{Pw}=$ pterygoid wedge. Copyright Ariel Kaen. Published with permission. Figure is available in color online only.

endonasal transpterygoid approach, especially in patients with poorly pneumatized sinuses..$^{10}$

The VN travels through the pterygoid or vidian canal (VC) in the sphenoid bone, so it can be localized during the initial steps of the endoscopic endonasal approach. Hence, dissection of the VN from its opening in the sphenoid bone naturally leads to the anterior genu of the petrous ICA. ${ }^{10}$ Many publications focus on different anatomical landmarks of the anterior end of the $\mathrm{VC}$ and how the endonasal transpterygoid approach may be performed safely. ${ }^{3-5,16,17,19,23}$ However, the literature is lacking a thorough anatomical description of the posterior and inferior limits of the endonasal transpterygoid avenue. Although the identification of the $\mathrm{VN}$ in the proximal aspect of its canal may permit us to safely identify the anterior genu of the ICA, the endoscopic anatomy of the most inferior and posterior area surrounding the ICA in this region-that is, the deepest and most posterior limit of the endoscopic endonasal transpterygoid route-has not been well documented..$^{11,12}$

Accordingly, the main goal of this study is to detail and quantify the anatomical relationship between the posterior end of the $\mathrm{VN}$, the posterior end of the $\mathrm{VC}$, and the neuro-fibrocartilaginous components of the FL and highlight how such peri-lacerum anatomy is crucial for safe endoscopic endonasal transpterygoid surgery.

\section{Methods}

\section{Anatomical Dissections}

Eight human anatomical specimens (16 sides) were used for this study. All specimens were injected with red and blue silicone via the ICAs and internal jugular veins, respectively. The surgical dissections were performed using paranasal sinus and skull base/neurosurgical endoscopic instruments (Karl Storz) and a high-speed drill with an angled handpiece as well as diamond cutting burrs (Medtronic Inc.). The dissections were achieved via a pure endonasal endoscopic approach. Visualization was accomplished with rod-lens endoscopes (4-mm diameter, $18-\mathrm{cm}$ length) with $0^{\circ}, 30^{\circ}$, and $45^{\circ}$ lenses (Karl Storz Endoscopy; Karl Storz), coupled to a high-definition camera and video monitor. Both video and standard digital images were obtained during dissections using the AIDA recording system (Karl Storz).

\section{Endoscopic Endonasal Transpterygoid Approach}

An endoscopic endonasal transpterygoid approach was performed as described in the pertinent literature. ${ }^{2,6,7,9,18-21}$

The surgical corridor was created by removing both middle turbinates and resecting the posterior nasal septum in order to create conditions that allow use of the endoscope and instruments through both nostrils. The medial wall of the maxillary sinus was removed to gain access to its posterior wall, which constitutes the anterior wall of the pterygopalatine fossa, and to the sphenopalatine foramen, through which the sphenopalatine artery reaches the nasal cavity; inferior turbinectomy and removal of the lateral nasal wall down to the level of the nasal floor were performed as well.

The orbital process of the palatine bone was removed, and the sphenopalatine foramen was enlarged to expose the anterior portion of the pterygoid process. The posterior wall of the maxillary sinus was dissected laterally, and the pterygopalatine fossa contents were displaced laterally as well (Fig. 1).

After performing the initial part of the endoscopic endonasal transpterygoid approach, dissection proceeds in a stepwise fashion, according to the following 3 main steps.

\section{Step 1: Exposure of the Anterior End of the VC}

The anterior end of the VC is located inferomedially to the foramen rotundum, on the upper medial part of the pterygoid process anterior surface (Fig. 2A), which opens into the medial part of the pterygopalatine fossa posterior wall.

After endoscopic endonasal exposure of the pterygopalatine fossa, the anterior edge of the $\mathrm{VC}$ can be observed. After lateral transposition of the neurovascular compartment of the pterygopalatine fossa, the $\mathrm{VN}$ can be isolated and followed posteriorly inside its canal, which is located at the intersection of the lateral wall of the sphenoid and the medial pterygoid plate. 

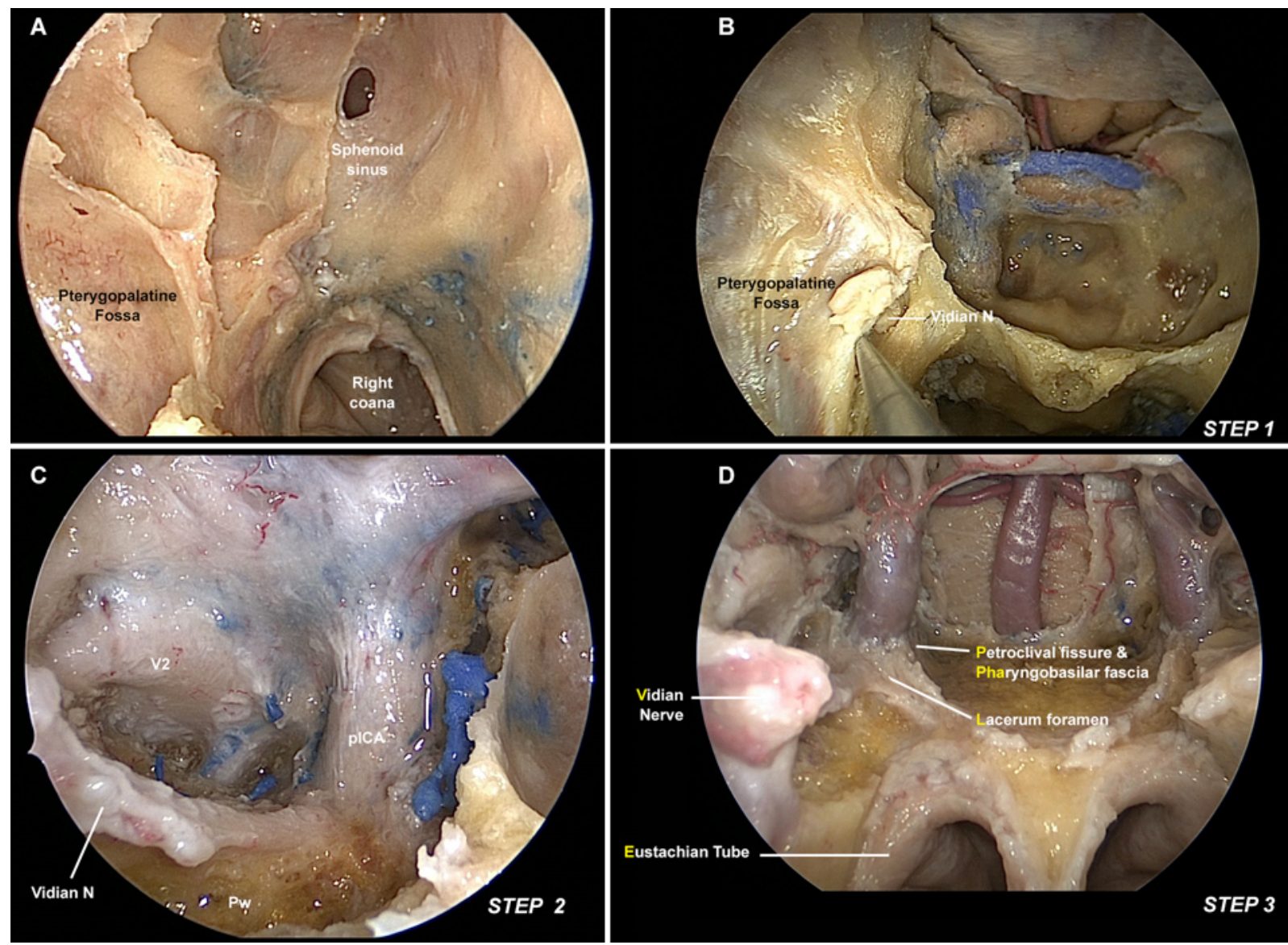

FIG. 2. Endoscopic endonasal transpterygoid approach. Stepwise dissection to the VELPPHA area. The main anatomical landmarks in the right nasal cavity are shown (A). In step 1, the anterior end of the VC is exposed after moving the pterygopalatine fossa content laterally (B). In step 2, the VC is followed in an anterior to posterior direction in order to reach the FL (C). In step 3 , the VELPPHA area is exposed and the surrounding neurovascular and ligamentous structures are visible (D). Copyright Ariel Kaen. Published with permission. Figure is available in color online only.

In our stepwise dissection, the identification of the most anterior part of the VC represents step 1 (Fig. 2B).

\section{Step 2: Follow the VC}

In step 2 of our dissection, the $\mathrm{VC}$ is followed with the aim of reaching the posterior end of the endonasal transpterygoid approach.

The dissection proceeds with drilling of the pterygoid process base around the $\mathrm{VC}$ in an anterior to posterior direction to expose the fibrocartilaginous area surrounding the foramen lacerum (FL). Specifically, the circumferential drilling of the $\mathrm{VC}$ starts in the inferomedial quadrant and then moves on to the infravidian segment.

The inferior remnants of the pterygoid plates are removed in order to expose the eustachian tube (ET). Muscles related to the ET, namely the tensor veli palatini and the levator veli palatini muscles, can then be visualized. Once the depth of the VC is clearly determined, drilling is performed in the most superolateral aspect of the canal. The clivus and the petrous apex are progressively drilled from medial to lateral, and the FL and the anterior genu of the ICA are seen, bound together by fibrous tissue. This second step is finished once the bone of the $\mathrm{VC}$ has been completely drilled out (Fig. 2C).

\section{Step 3: Posterior End of the VC-the VELPPHA Area}

In the last step of our dissection, the region that we refer to as the VELPPHA area is exposed. This area represents the posterior limit of the transpterygoid approach, and it is formed by multiple cartilaginous fibers that surround the petrous segment of the ICA at the level of the FL. Hence, during this third step of our dissection, the key anatomical relationships between the VC, ET, FL, petroclival fissure, and pharyngobasilar fascia can be identified (Fig. 2D).

\section{Quantitative Assessment and Statistical Analysis}

The anteroposterior lengths of the $\mathrm{VN}$ and $\mathrm{VC}$ were quantified after endoscopic endonasal dissections. Measurements were obtained in 3 different planes as follows in order to evaluate the working space: $\mathrm{FL}_{1}$, the distance between the posterior end of the $\mathrm{VC}$ and the posterior edge of the lacerum ICA in the axial plane; $\mathrm{FL}_{2}$, the distance between the $\mathrm{VN}$ at the petrous ICA and a vertical line parallel to the lacerum ICA in the coronal plane; and $\mathrm{FL}_{3}$, the distance between the anterior genu of the petrous ICA and the eustachian tube [ET] in the craniocaudal plane. The navigation system (Medtronic Inc.) showed the point coordinates and was used for measuring the distances (Fig. 


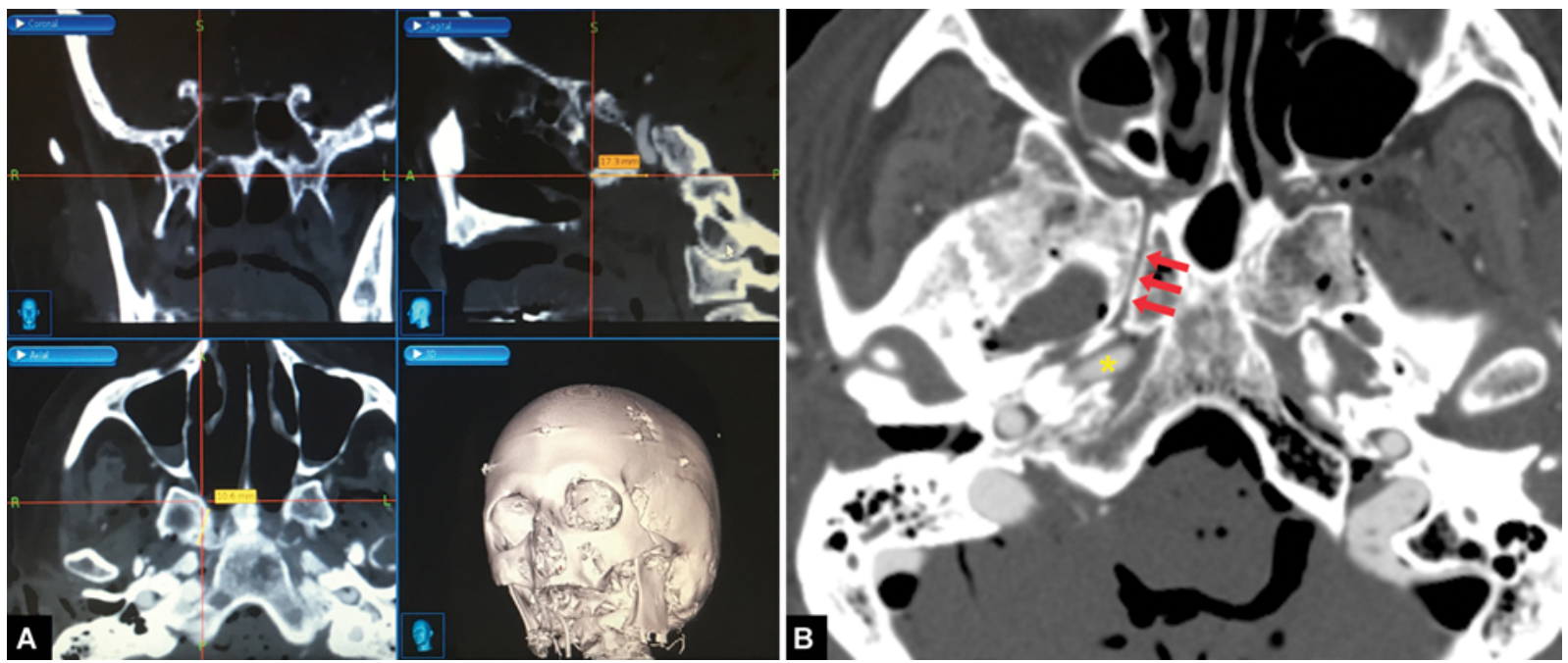

FIG. 3. A: Image-guidance screenshots depicting the main quantitative measurement obtained. B: Axial CT images were used to calculate VC length (red arrows) at the level of the petrous ICA (yellow asterisk). Figure is available in color online only.

3). The distances were measured by a simple mathematical method (straight distance between 2 coordinates). The volume was obtained by means of the following formula: $\left(\left[\mathrm{FL}_{1}\right] \times\left[\mathrm{FL}_{2}\right] \times\left[\mathrm{FL}_{3}\right]\right) / 2$. Statistical analyses were performed with SPSS version 22 software (IBM Corp.). Quantitative variables (including distances) were expressed as means and standard deviations. The Student t-test was used to determine the statistical significance of differences between right and left distances $(\mathrm{p}<0.05$ was considered statistically significant).

\section{Results}

\section{Detailed Description of the VELPPHA Area Components}

Our dissections show that there are 5 main anatomical structures surrounding the anterior genu of the petrous ICA in the posterior limit of the endoscopic endonasal transpterygoid approach: the end of the vidian canal (VC), the eustachian tube (ET), the foramen lacerum (FL), the petroclival fissure, and the pharyngobasilar fascia. We designed a novel acronym with the aim of underscoring the importance of the described anatomical structures that represent the posterior limit of the endoscopic endonasal transpterygoid approach-namely VELPPHA (with $\mathrm{V}$ standing for vidian canal, $\mathrm{E}$ for eustachian tube, $\mathrm{L}$ for foramen lacerum, $\mathrm{P}$ for petroclival fissure, and PHA for pharyngobasilar fascia). Each single component of the VELPPHA area is described in detail below. This area is formed by multiple cartilaginous fibers adhering to the $\mathrm{VC}$, the ET, the inferior part of the FL, the petroclival fissure, and the pharyngobasilar fascia. All these structures are below the horizontal segment of the petrous ICA. Of utmost importance, no neurovascular structures are observed within the VELPPHA area, thus making it a safe zone for surgery in the posterior end of the endoscopic endonasal transpterygoid approach (Video 1).

VIDEO 1. Refining the anatomical boundaries of the transpterygoid approach: the VELPPHA area concept. The transpterygoid approach is the usual route to access the petrous apex, petroclival fissure, Meckel's cave, or cavernous sinus. The vidian canal (VC) is a critical surgical landmark to localize the anterior genu of the horizontal segment of the petrous ICA. The VC connects the pterygopalatine fossa and the foramen lacerum (FL) and has 3 parts. 1) The anterior opening of the VC is located on the anterior surface of the pterygoid process. 2) The canal goes from anterior to posterior in a straight course with a trumpet shape, open toward the front. 3) The posterior opening of the VC is filled with cartilaginous tissue from 5 different anatomical structures surrounding the anterior genus of the petrous ICA. VELPPHA is an acronym for the combination of the following anatomical structures: the posterior end of the VC, the eustacian tube (ET), the foramen lacerum (FL), the petroclival fissure, and the pharyngobasilar fascia. The transpterygoid approach can be classified in 3 steps. Step 1: The first step is to look for the anterior edge of the VC that is usually hidden behind the pterygopalatine fossa. In the early dissection of the right nasal fossa in this video clip, one can see why we need to open the pterygopalatine fossa to access the anterior end of the VC. In the more advanced endoscopic dissection view, the dissector pushes the pterygopalatine contents laterally and identifies the medial pterygoid "wedge" where the vidian nerve (VN) is located. Step 2: Once the anterior end of the VN is identified, we can move to the second step, "follow the vidian canal." We start drilling the inferior and medial aspect of the VC, then the inferolateral quadrant. The "infravidian" bone is a safe drilling area throughout the extent of the VC. Considering the VC as a clockface, drilling of the right VC should be initiated from the 3 to 6 o'clock position. In this sequential dissection where the bone is removed along the inferior and medial aspect of the VC, the depth of the pterygoid wedge helps us to understand where the VC ends. Step 3: After the drilling of the VC bone is completed, we move on to step 3. The VELPPHA area represents the posterior limit of the transpterygoid approach and is formed by multiple cartilaginous fibers adhering to the VC, the ET, the inferior part of the foramen lacerum, the petroclival fissure, and the pharyngobasilar fascia. In this advanced dissection we can identify the 3 different fibers of the VELPPHA ligament: anterior, medial, and posterior. It should be noted that all these structures are below the horizontal segment of the petrous ICA. Copyright Ariel Kaen. Published with permission. Click here to view.

\section{Vidian Nerve and Vidian Canal}

The vidian nerve $(\mathrm{VN})$ is formed by the union of the greater petrosal nerve and the deep petrosal nerve. It is located on the superior surface of the most medial segment of the horizontal petrous of the ICA, and if followed 
TABLE 1. Measurements of the VN, VC, and FL

\begin{tabular}{lccc}
\hline Measurement & Right & Left & p Value \\
\hline $\mathrm{VN}, \mathrm{mm}$ & $23.12(1.95)$ & $23.90(1.45)$ & 0.209 \\
\hline $\mathrm{VC}, \mathrm{mm}$ & $14.18(1.05)$ & $13.9(0.85)$ & 0.413 \\
\hline $\mathrm{FL}_{1}, \mathrm{~mm}$ & $6.1(2)$ & $5.9(2.1)$ & 0.784 \\
\hline $\mathrm{FL}_{2}, \mathrm{~mm}$ & $5.8(1.25)$ & $5.6(1.1)$ & 0.634 \\
\hline $\mathrm{FL}_{3}, \mathrm{~mm}$ & $8.4(0.55)$ & $8.3(0.55)$ & 0.610 \\
\hline $\mathrm{FL}$ vol, $\mathrm{mm}^{3}$ & $99(63.3)$ & $91(56.6)$ & 0.708 \\
\hline
\end{tabular}

$\mathrm{FL}=$ foramen lacerum; $\mathrm{FL}_{1}=\mathrm{FL}$ in axial plane; $\mathrm{FL}_{2}=\mathrm{FL}$ in coronal plane; $\mathrm{FL}_{3}=$ $\mathrm{FL}$ in craniocaudal plane; $\mathrm{VC}=$ vidian canal; $\mathrm{VN}=$ vidian nerve.

Data are mean (SD).

from its origin, it courses from posterior to anterior and from superior to inferior through the cartilaginous tissue in the upper part of the anterolateral margin of the FL to enter the posterior end of the VC. The proximal portion of the $\mathrm{VN}$ is encased by the fibrocartilaginous tissue that forms the lower portion of the FL; a complex set of vertical fibers of the FL encloses the nerve before it reaches the posterior end of the VC. The anteroposterior length of the VN averaged $23.12 \mathrm{~mm}$ (range $21.1-25.0 \mathrm{~mm}$ ) on the right and $23.90 \mathrm{~mm}$ (range $22.0-24.9 \mathrm{~mm}$ ) on the left (Table 1 and Fig. 3).

On the other hand, the VC is located inside the base of the pterygoid process and connects the FL with the pterygopalatine fossa. It has a slightly curved pathway, which is directed posterolaterally when seen from an endonasal view. Accordingly, when the $\mathrm{VC}$ is reached from the endonasal perspective, its anterior opening is placed in a most medial position when compared with its posterior end. The posterior opening of the VC was situated in all dissections superior to the ET and below the horizontal petrous ICA. The anteroposterior length of the VC averaged $14.18 \mathrm{~mm}$ (range 13.0-15.1 mm) on the right and $13.90 \mathrm{~mm}$ (range 12.9-14.6 mm) on the left (Table 1 and Fig. 4).

\section{Eustachian Tube, Petroclival Fissure, and Pharyngobasilar Fascia}

The eustachian tube (ET), with its cartilaginous portion and torus tubarius, is in a strict relationship with the posterior border of the medial pterygoid process anteriorly, while it is attached to the clival region and FL posteriorly. Multiple vertical cartilaginous fibers strongly attach the superior and medial border of the ET to the lacerum area. These fibers are longer in the medial portion and very short in the lateral region (Fig. 5). In this triangular space, namely the supratubaric space, no anatomical or vascular structure was observed in the cadaveric dissections.

The petroclival fissure, which sits along the lateral edge of the clivus, separates the occipital bone and the petrous part of the temporal bone. This fissure extends from the FL above up to the jugular foramen below. The inferior petrosal sinus runs along the petroclival fissure on the intracranial surface. Horizontal cartilaginous fibers join the petrous part of the temporal bone to the occipital and sphenoid bones. These fibers are more extensive in the FL and decrease progressively toward the jugular foramen (Fig. 6).

The pharyngobasilar fascia, also known as the pharyngeal aponeurosis, originates from the pharyngeal tubercle and runs laterally across the clivus to the petrous temporal bone, immediately anterior to the carotid foramen, and then passes anteromedially to reach its insertion along the cartilaginous ET and the posterior edge of the medial pterygoid plate, at the level of the hamulus. This aponeurosis represents a horizontal fiber as we observed on the
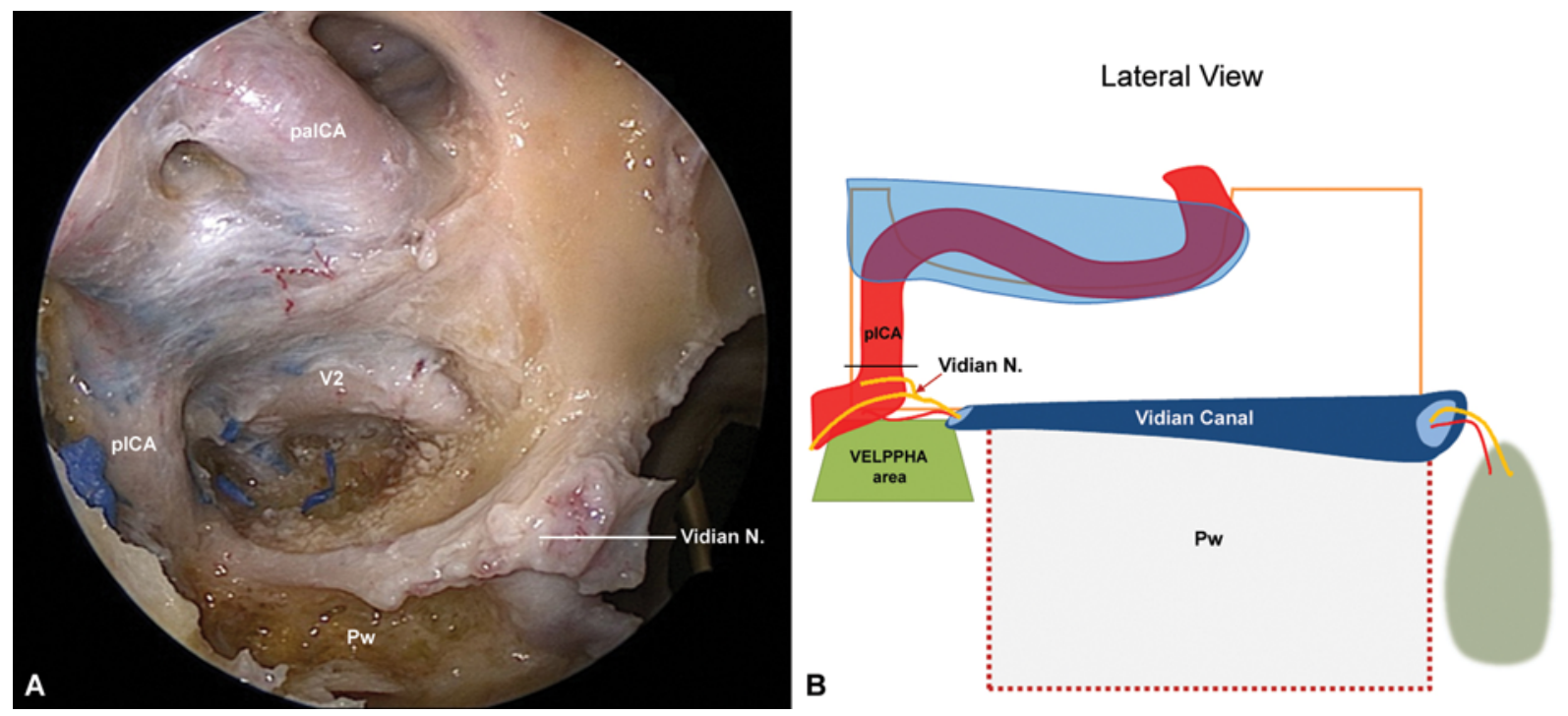

FIG. 4. A: $A 45^{\circ}$ endoscopic view of the endonasal transpterygoid cadaveric dissection showing the VN going from the pterygopalatine fossa to the FL. Due to the trajectory from medial to lateral and from inferior to superior, in the posterior limits of the transpterygoid approach, the nerve crosses in front of the horizontal portion of the carotid and is then placed on top. B: Lateral schematic illustration facilitating understanding of the posterior limit of the VC along the pterygoid wedge. The VN passes along the anterior half of the upper surface of the horizontal segment of the ICA before turning downward along the anterior surface of the artery to reach the FL. paICA = parasellar ICA; pICA = paraclival ICA; Pw = pterygoid wedge. Copyright Ariel Kaen. Published with permission. Figure is available in color online only. 


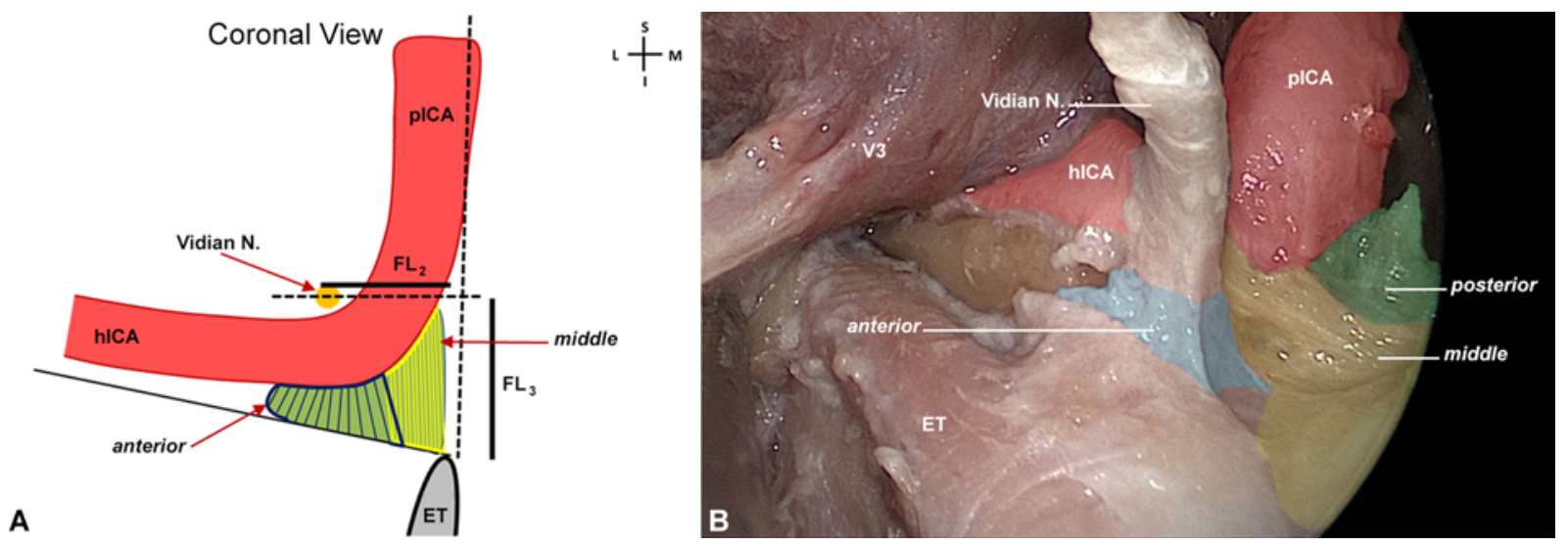

FIG. 5. A: Coronal illustration view showing how the vertical fibers of the VELPPHA area attach to the ET, especially the medial ones (yellow). B: A $45^{\circ}$ endoscopic view showing the course of the $\mathrm{VN}$ and the close relationship with the horizontal petrous ICA. The anterior and medial fibers of the VELPPHA area come from the fibrocartilaginous part of the ET. For definitions of $\mathrm{FL}_{2}$ and $\mathrm{FL}_{3}$, refer to the Quantitative Assessment and Statistical Analysis subsection of Methods. hICA = horizontal petrous ICA; $p I C A=$ paraclival ICA. Copyright Ariel Kaen. Published with permission. Figure is available in color online only.

petroclival fissure, but less hard and more superficial when we work from the nose.

\section{Foramen Lacerum}

The foramen lacerum (FL) is bound anteriorly by the junction of the body, lingular process of the greater sphenoid wing, and adjoining roots of the pterygoid wedge of the sphenoid bone; posterolaterally by the petrous apex of the temporal bone; and medially by the clival portion of the occipital bone. The petrous ICA, after exiting the petrous apex, occupies the upper part of the FL. The anterior genu of the ICA sits above the anterior half and does not pass through the foramen.

The anatomical measurements of the FL were as follows: $\mathrm{FL}_{1}$ averaged $6.1 \mathrm{~mm}$ (range $4.2-8.4 \mathrm{~mm}$ ) on the right and $5.9 \mathrm{~mm}$ (range $4.0-8.2 \mathrm{~mm}$ ) on the left; $\mathrm{FL}_{2}$ averaged $5.8 \mathrm{~mm}$ (range $4.4-6.9 \mathrm{~mm}$ ) on the right and 5.6 $\mathrm{mm}$ (range $4.2-6.4 \mathrm{~mm}$ ) on the left; $\mathrm{FL}_{3}$ averaged $8.4 \mathrm{~mm}$ (range 8.0-9.1 mm) on the right and $8.3 \mathrm{~mm}$ (range 7.9-9.0 $\mathrm{mm})$ on the left; and the total volume averaged $99 \mathrm{~mm}^{3}$ (range 49.2-175.8 $\mathrm{mm}^{3}$ ) on the right and $91 \mathrm{~mm}^{3}$ (range 44.2-157.4 $\mathrm{mm}^{3}$ ) on the left (Table 1). (See the Quantitative Assessment and Statistical Analysis subsection of Methods for definitions of $\mathrm{FL}_{1}, \mathrm{FL}_{2}$, and $\mathrm{FL}_{3}$.)

The dense fibrocartilaginous tissue of the FL presents 4 different fibrous attachments, simulating skull base ligaments (i.e., vertical ligaments and horizontal ligaments) (Fig. 7). Three vertical ligaments can be identified: 1) the anterior ligament between the anterior lip of the FL and the posterior wall of the pterygoid wedge, covering the
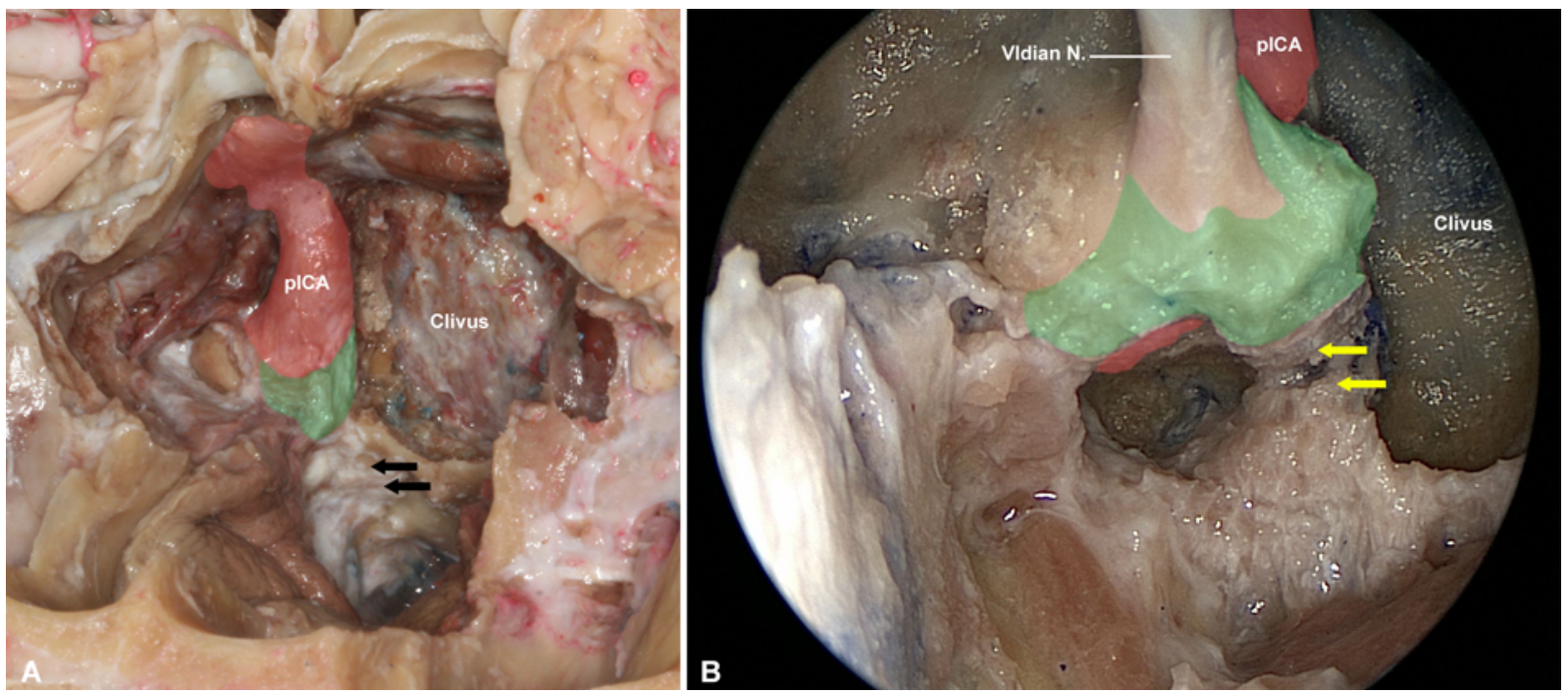

FIG. 6. A: The lacerum anatomy shown in a formalin-fixed cadaveric head. The VELPPHA area (green zone) presents a group of fibers that are distributed horizontally to the FL. The most superficial fibers come from the pharyngobasilar fascia (black arrows), while the deeper fibers originate in the petroclival fissure. B: An endoscopic view of the completely dissected petroclival fissure showing the posterior horizontal fibers of the VELPPHA area attached to the FL in a deeper plane (yellow arrows). pICA = paraclival ICA. Copyright Ariel Kaen. Published with permission. Figure is available in color online only. 

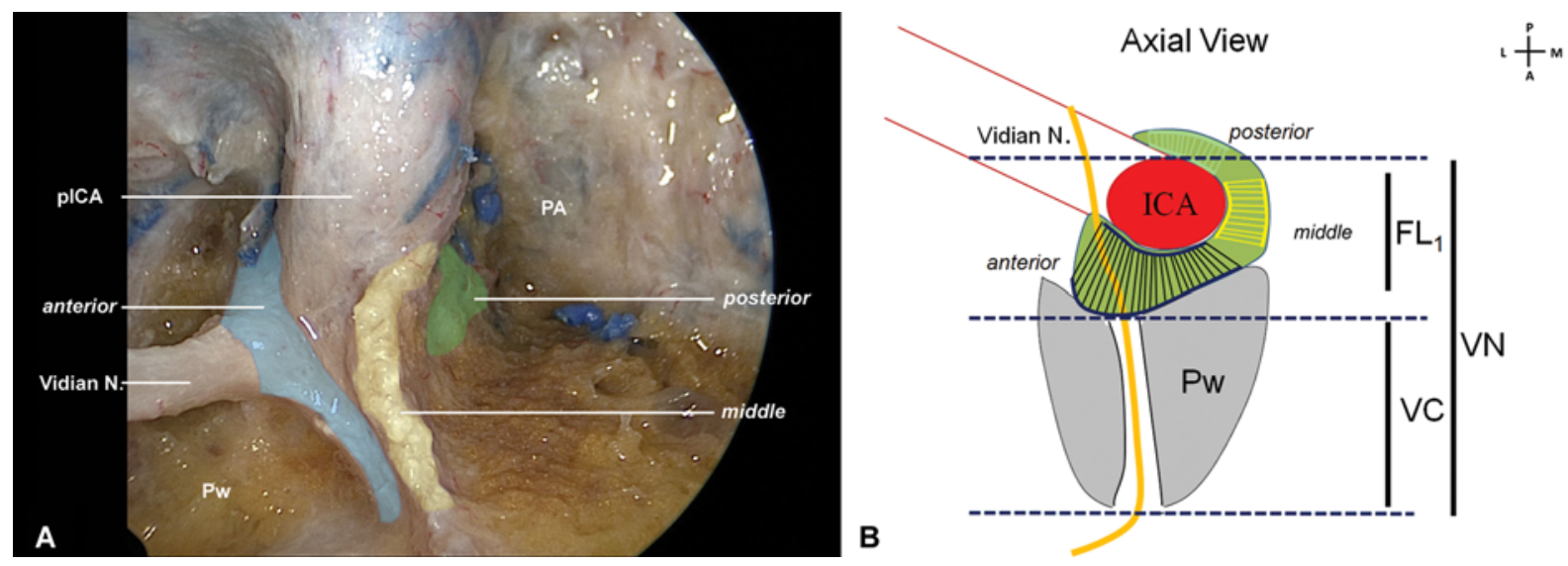

FIG. 7. A: $A 45^{\circ}$ endoscopic view of the pICA. It can be observed how the $\mathrm{VN}$ is surrounded by the anterior fibers (blue) that extend from the FL toward the pterygoid wedge. The medial fibers (yellow) extend toward the ET, and finally, the posterior fibers adhere to the posterior border of the FL with the union of the petrous apex. B: An axial illustration view of the vertical fibers showing the 3 different attachments: the pterygoid wedge, the eustachian tube, and the petrous apex. PA = petrous apex; $P w=$ pterygoid wedge. Copyright Ariel Kaen. Published with permission. Figure is available in color online only.

$\mathrm{VN}$ before it enters the posterior end of the $\mathrm{VC} ; 2)$ the middle ligament located from the anterior lip of the FL to the superior portions of the ET, reaching the FL between the body of the sphenoid sinus and the pterygoid wedge; and 3) the posterior ligament (the smallest of the vertical fibers), which extends from the posterior lip of the FL to the most medial and superior segment of the petroclival synchondrosis.

Finally, horizontal ligaments are part of the fibrocartilaginous fibers that go from the medial to the lateral area of the FL. These ligaments have 2 layers, a superficial one coming from the pharyngobasilar fascia and another that originates from the petroclival fissure.

During the transpterygoid approach, we remove most of the pterygoid wedge around the $\mathrm{VC}$ following the $\mathrm{VN}$. However, the diamond drill stops prematurely in the posterior end of the $\mathrm{VC}$ in a dense fibrocartilaginous tissue.

\section{Discussion}

Recent advancement in endoscopic endonasal skull base surgery has widened the target of the technique to the coronal plane approaches described as Kassam zones., ${ }^{4,10,19,23}$ A comprehensive transpterygoid approach involves identification and exposure of the cavernous, paraclival, and horizontal segments of the ICA (lacerum and petrous). ${ }^{8,9,13}$ All these neurovascular structures usually require a transpterygoid route. The anterior genu of the ICA, the bend where the horizontal segment turns upward around the medial edge of the petrous apex to end in the anterior vertical segment, is inferomedial to the trigeminal nerve and Gasserian ganglion, above the foramen lacerum (FL), and in the lateral wall of the sphenoid sinus. ${ }^{16}$ The anterior genu and anterior vertical segment are referred to as the lacerum segment by Bouthillier et al. ${ }^{1}$ Many studies have reported how to find the anterior end of the $\mathrm{VC}$ and follow the $\mathrm{VN}$ toward the lacerum segment of the petrous horizontal ICA. ., $^{3,10,15,16,19}$ The VC is a useful landmark to reach the lacerum segment of the ICA at its transition from the horizontal petrous segment to its vertical paraclival segment, especially in patients with poor sphenoid sinus pneumatization or those with recurrent pathology in whom the conventional surgical anatomy may be distorted. Moreover, exposure of the VN allows further exposure of the pterygoid base and lateral wall of the sphenoid sinus, as part of the transpterygoid approach. In the transnasal endoscopic procedure, the VN can be followed backward from the pterygopalatine fossa under a prominence over the nerve in the floor of the sphenoid sinus to the area above the FL and the lower edge of the anterior genu.

However, even if it is well accepted in the pertinent literature, care should be taken when dealing with the concept of "following the vidian nerve" and considering it the main anatomical landmark to check for petrous ICA position. ${ }^{10-12,15}$

The so-called VELPPHA area (for vidian nerve, eustachian tube, foramen lacerum, petroclival fissure, and pharyngobasilar fascia) is a group of skull base fibrocartilaginous tissues that join together and represent an easy anatomical landmark at the posterior limits of the endonasal transpterygoid approach.

In this contribution we detailed and showed a stepwise dissection aimed at reaching the VELPPHA area. The technique herewith described includes drilling medially and inferiorly to the VC; afterwards dissection proceeds in a posterior direction until a fibrocartilaginous tissue is identified, which marks the attachment to the FL and the anterior border of the second genu of the ICA. ${ }^{11,12,14}$ Moving in a medial to lateral direction along the anteroinferior border of the horizontal ICA, the anterior genu of the ICA can be exposed after removal of the cartilaginous ET. Recently Taniguchi et al..$^{22}$ described the supra-eustachian triangle, a space bordered by the inferior aspect of the horizontal segment of the petrous ICA, by the superior aspect of the $\mathrm{ET}$, and inferiorly by a vertical line along the medial aspect of the lacerum ICA. However, to access this triangular space, it is mandatory to identify the posterior border of the transpterygoid approach. Accordingly, the VELPPHA area represents not only the "safe area" located at the posterior limit of the transpterygoid approach but also the content 
of the supra-eustachian triangle. Oakley et al. ${ }^{14}$ define the vidian-eustachian junction as a secure area in which to find the ICA. Unfortunately, using the VN as a guide in the posterior limits of the transpterygoid approach, we may not be able to totally identify a safe anatomical landmark, since the nerve usually crosses the anterosuperior surface of the horizontal portion of the ICA.

Hence, in our dissections we described the fibrocartilaginous complex that fills the lower portion of the FL as a set of intercrossed fibers that mainly form 3 vertical beams and 1 horizontal beam. We are aware of the limitations of these dissections, given the strong adherence of these fibers to each other and the enormous intercrossing of fibers that prevents the proper categorization of the whole. However, the described ligaments represent the most consistent portions in the dissected specimens as well as a set of fibers with a constant and recognizable direction. We have observed that the fibers that represent the vertical medial ligament coming from the ET are especially vigorous and can be recognized early when drilling the $\mathrm{VC}$, always below the VN plane, and they anticipate the VELPPHA area.

Dissection of this area is difficult due to the strong attachments, but in most clinical cases we only need to know where the posterior limit of our approach is, rather than needing to enter the VELPPHA area. Accordingly, we may refine the "classic" concept from "following the vidian nerve" to "following the vidian canal" from anterior to posterior (endonasal view); therefore, the transpterygoid approach may end when the VELPPHA area (transition between bone and dense fibrocartilaginous tissue) is encountered. The VELPPHA area is an important anatomical landmark if there is the need to displace the petrous ICA (at the level of the FL). No nervous or vascular structures pass through the FL. Hence, it is safe to separate the lacerum segment of the cartilaginous ET from the FL by transecting the soft tissue just below the level of the VC.

\section{Conclusions}

The concept of the VELPPHA area is described in this anatomical report to further advance the knowledge of the endoscopic endonasal transpterygoid approach. The area herewith described represents the posterior limit of the transpterygoid route and, given that it is free of neurovascular structures, it could be thought as a safe zone for surgery in the posterior end of the endoscopic endonasal transpterygoid approach. Early identification of the VELPPHA area can contribute to safely performing such an endoscopic endonasal corridor approach expanded to the lateral aspect of the skull base, especially in patients with poorly pneumatized sphenoid sinuses.

\section{Acknowledgments}

We thank Fatima Iarlori for the artistic drawings that she created for this paper.

\section{References}

1. Bouthillier A, van Loveren HR, Keller JT: Segments of the internal carotid artery: a new classification. Neurosurgery 38:425-433, 1996

2. Cavallo LM, Messina A, Gardner P, Esposito F, Kassam AB,
Cappabianca P, et al: Extended endoscopic endonasal approach to the pterygopalatine fossa: anatomical study and clinical considerations. Neurosurg Focus 19(1):E5, 2005

3. Dolci RL, Upadhyay S, Ditzel Filho LF, Fiore ME, Buohliqah L, Lazarini PR, et al: Endoscopic endonasal study of the cavernous sinus and quadrangular space: anatomic relationships. Head Neck 38 (Suppl 1):E1680-E1687, 2016

4. Falcon RT, Rivera-Serrano CM, Miranda JF, Prevedello DM, Snyderman CH, Kassam AB, et al: Endoscopic endonasal dissection of the infratemporal fossa: anatomic relationships and importance of eustachian tube in the endoscopic skull base surgery. Laryngoscope 121:31-41, 2011

5. Fortes FS, Pinheiro-Neto CD, Carrau RL, Brito RV, Prevedello DM, Sennes LU: Endonasal endoscopic exposure of the internal carotid artery: an anatomical study. Laryngoscope 122:445-451, 2012

6. Fortes FS, Sennes LU, Carrau RL, Brito R, Ribas GC, Yasuda A, et al: Endoscopic anatomy of the pterygopalatine fossa and the transpterygoid approach: development of a surgical instruction model. Laryngoscope 118:44-49, 2008

7. Freeman JL, Sampath R, Quattlebaum SC, Casey MA, Folzenlogen ZA, Ramakrishnan VR, et al: Expanding the endoscopic transpterygoid corridor to the petroclival region: anatomical study and volumetric comparative analysis. J Neurosurg 128:1855-1864, 2018

8. Hosseini SM, Razfar A, Carrau RL, Prevedello DM, Fernandez-Miranda J, Zanation A, et al: Endonasal transpterygoid approach to the infratemporal fossa: correlation of endoscopic and multiplanar CT anatomy. Head Neck 34:313-320, 2012

9. Kasemsiri P, Solares CA, Carrau RL, Prosser JD, Prevedello DM, Otto BA, et al: Endoscopic endonasal transpterygoid approaches: anatomical landmarks for planning the surgical corridor. Laryngoscope 123:811-815, 2013

10. Kassam AB, Vescan AD, Carrau RL, Prevedello DM, Gardner P, Mintz AH, et al: Expanded endonasal approach: vidian canal as a landmark to the petrous internal carotid artery. $\mathbf{J}$ Neurosurg 108:177-183, 2008

11. Liu J, Pinheiro-Neto CD, Fernandez-Miranda JC, Snyderman $\mathrm{CH}$, Gardner PA, Hirsch BE, et al: Eustachian tube and internal carotid artery in skull base surgery: an anatomical study. Laryngoscope 124:2655-2664, 2014

12. Liu J, Sun X, Liu Q, Wang D, Wang H, Ma N: Eustachian tube as a landmark to the internal carotid artery in endoscopic skull base surgery. Otolaryngol Head Neck Surg 154:377-382, 2016

13. Muto J, Prevedello DM, Ditzel Filho LF, Tang IP, Oyama K, Kerr EE, et al: Comparative analysis of the anterior transpetrosal approach with the endoscopic endonasal approach to the petroclival region. J Neurosurg 125:1171-1186, 2016

14. Oakley GM, Ebenezer J, Hamizan A, Sacks PL, Rom D, Sacks R, et al: Finding the petroclival carotid artery: the vidian-eustachian junction as a reliable landmark. J Neurol Surg B 79:361-366, 2018

15. Osawa S, Rhoton AL Jr, Seker A, Shimizu S, Fujii K, Kassam AB: Microsurgical and endoscopic anatomy of the vidian canal. Neurosurgery 64 (5 Suppl 2):385-412, 2009

16. Osawa S, Rhoton AL Jr, Tanriover N, Shimizu S, Fujii K: Microsurgical anatomy and surgical exposure of the petrous segment of the internal carotid artery. Neurosurgery 63 (4 Suppl 2):210-239, 2008

17. Pinheiro-Neto CD, Fernandez-Miranda JC, Prevedello DM, Carrau RL, Gardner PA, Snyderman CH: Transposition of the pterygopalatine fossa during endoscopic endonasal transpterygoid approaches. J Neurol Surg B Skull Base 74:266-270, 2013

18. Pinheiro-Neto CD, Fernandez-Miranda JC, Rivera-Serrano CM, Paluzzi A, Snyderman CH, Gardner PA, et al: Endoscopic anatomy of the palatovaginal canal (palatosphenoidal 
canal): a landmark for dissection of the vidian nerve during endonasal transpterygoid approaches. Laryngoscope 122:612,2012

19. Prevedello DM, Pinheiro-Neto CD, Fernandez-Miranda JC, Carrau RL, Snyderman CH, Gardner PA, et al: Vidian nerve transposition for endoscopic endonasal middle fossa approaches. Neurosurgery 67 (2 Suppl Operative):478-484, 2010

20. Rivera-Serrano CM, Terre-Falcon R, Fernandez-Miranda J, Prevedello D, Snyderman CH, Gardner P, et al: Endoscopic endonasal dissection of the pterygopalatine fossa, infratemporal fossa, and post-styloid compartment. Anatomical relationships and importance of eustachian tube in the endoscopic skull base surgery. Laryngoscope 120 (Suppl 4):S244, 2010 (Abstract)

21. Solari D, Magro F, Cappabianca P, Cavallo LM, Samii A, Esposito F, et al: Anatomical study of the pterygopalatine fossa using an endoscopic endonasal approach: spatial relations and distances between surgical landmarks. J Neurosurg 106: $157-163,2007$

22. Taniguchi M, Akutsu N, Mizukawa K, Kohta M, Kimura H, Kohmura E: Endoscopic endonasal translacerum approach to the inferior petrous apex. J Neurosurg 124:1032-1038, 2016

23. Youssef A, Carrau RL, Tantawy A, Ibraheim A, Solares AC, Otto BA, et al: Endoscopic versus open approach to the infratemporal fossa: a cadaver study. J Neurol Surg B Skull Base 76:358-364, 2015

\section{Disclosures}

The authors report no conflict of interest concerning the materi- als or methods used in this study or the findings specified in this paper.

\section{Author Contributions}

Conception and design: Kaen, Cárdenas Ruiz-Valdepeñas. Acquisition of data: Kaen, Cárdenas Ruiz-Valdepeñas. Analysis and interpretation of data: Kaen, Cárdenas Ruiz-Valdepeñas. Drafting the article: Kaen, Cárdenas Ruiz-Valdepeñas, Di Somma. Critically revising the article: Kaen, Cárdenas Ruiz-Valdepeñas, Di Somma. Reviewed submitted version of manuscript: Kaen, Cárdenas Ruiz-Valdepeñas, Di Somma. Approved the final version of the manuscript on behalf of all authors: Kaen. Statistical analysis: Kaen, Di Somma. Administrative/technical/material support: Kaen, Di Somma. Study supervision: all authors.

\section{Supplemental Information \\ Videos}

Video 1. https://vimeo.com/272751154.

\section{Correspondence}

Ariel Kaen: Hospital Universitario Virgen del Rocío, Sevilla, Spain.kaenariel@hotmail.com. 